\title{
Suprafibrillar structures of collagen, evidence for local organization and auxetic behaviour in architectures
}

\author{
Kate Patten ${ }^{*}$, Tim Wess \\ School of Optometry and Vision Sciences, Cardiff University, Cardiff, UK; ${ }^{*}$ Corresponding Author: PattenK@,cf.ac.uk
}

Received 2 May 2013; revised 2 June 2013; accepted 10 June 2013

Copyright (C) 2013 Kate Patten, Tim Wess. This is an open access article distributed under the Creative Commons Attribution License, which permits unrestricted use, distribution, and reproduction in any medium, provided the original work is properly cited.

\begin{abstract}
The suprafibrillar organisation of collagen rich tissues is the keystone to the diversity of resultant structures made from relatively similar materials. The local organisation between fibrils may be essential to suprafibril structures that are critical to functionality such as transparency in cornea, where specific lateral relationships between fibrils dictate optical properties. Here we show that corneal X-ray diffraction combined with mechanical strains to disrupt a specific suprafibrillar relationship between fibrils evidence and a coherent staggered axial relationship between collagen fibrils. The data also shows evidence for auxetic behavior of the collagen fibrils and reveals a $120 \mathrm{~nm}$ diffraction feature previously unreported in collagen tissues. The results show that suprafibrillar organisation can be an essential component in tissue architecture that has hitherto been ignored, but now must be considered in mechanical and structural models.
\end{abstract}

Keywords: Collagen Structure; XRD (X-Ray Diffraction); Suprafibrillar; Organisation; Auxetic

\section{INTRODUCTION}

The suprafibrillar arrangement of collagen fibrils is often regarded as being at most a lateral packing of local order; however, fibril architecture is a major contributor to the exquisite fidelity of tissue properties and the mechanisms for controlling fibril disposition is poorly understood. There is some indirect evidence from a number of sources that axial interfibrillar registration can occur in collagen [1], and fibrillin rich microfibrils have regions of staggered fibrils within an intact tissue [2]. This type of interaction has implications for the local registration, spatial relationship and mechanical performance of a tissue. In addition, it is critical to know if this is a factor in the modulation of axial data intensity for the modelling of individual collagen fibrillar structures from $\mathrm{X}$-ray diffraction data. Cornea is a unique collagen material reliant on interfibrillar interactions for its high almost perfect transparent properties and extraordinary mechanical resilience $[3,4]$. Here, the ability of the cornea to transmit at least $99 \%$ of incident light is due to the highly regulated sub optical fibrillar size and suprafibrillar organisation of the lamellae [5,6]. The factors that direct and maintain the interfibrillar relationship have been mostly reported on the lateral interaction between the fibrils [7].

There is tentative indirect evidence that a highly coherent specific axial interfibrillar organisation also exists between collagen fibrils within cornea. In small angle $\mathrm{X}$-ray diffraction studies of cornea, the commonly observed intensity of the meridional diffraction series has a first order intensity that is very weak [8], whereas in most fibrillar collagen rich tissues, the intensity of the diffraction series follows a pattern of strong odd and weak even diffraction peak intensities where the first and third meridional diffraction order of many collagen rich tissues are more intense that the rest of the observable meridional intensities. Such a pattern of intensity in the meridional orders is believed to derive from the well characterised $\sim 65 \mathrm{~nm}$ axial repeating step function of the gap and overlap regions that arises from the axial molecular packing of collagen molecule within the fibril the Hodge Petruska model [9] as demonstrated in the schematic in Figure 1.

The difference observed in the diffraction of cornea points to two possible modulations of this structural feature, it is due to either 1) the regular presence of proteogylcans along the fibril that modulates the axial electron density due to collagen axial packing [8], or 2) it is 

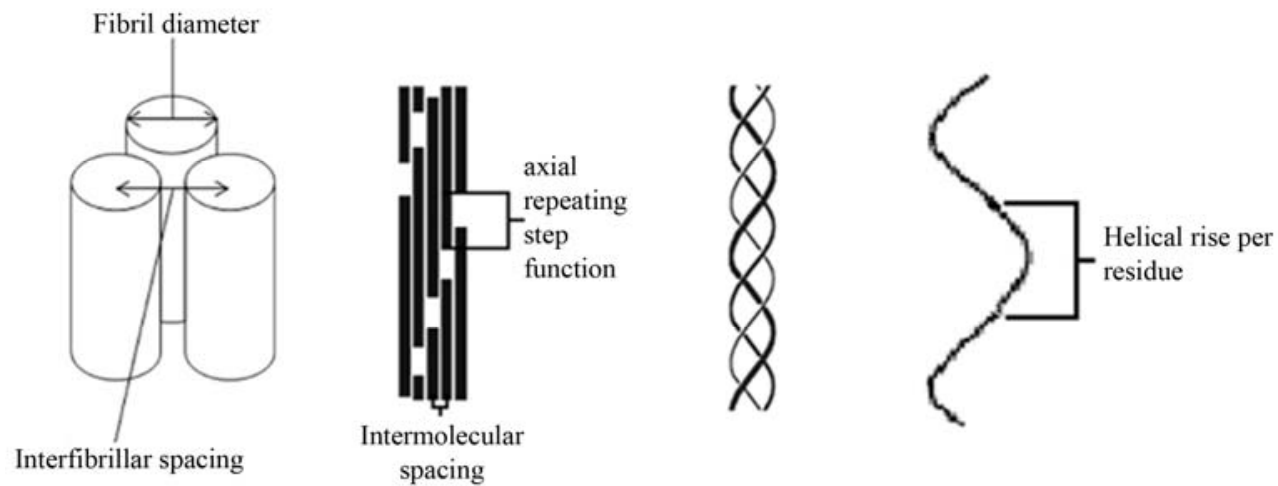

Figure 1. Schematic of the collagen hierarchy. From left to right, fibril packing, microfibril packing, collagen triple helix and helical rise per residue. Diagram is not to scale.

due to a coherent axial suprafibrillar relationship between fibrils that involves a specific axial spatial offset between neighbouring fibrils. Such interactions may be held in place by specific linkages dictated by proteoglycans that are known to be important in the interfibrillar relationships, their spacing, and the all important transparency $[5,10,11]$.

If the second proposition (a coherent axial relationship) here is correct, a possible way of probing the suprafibrillar organisation would be by mechanical extension of the sample whilst simultaneously recording small angle $\mathrm{X}$-ray fibre diffraction data to a point where the suprafibrillar coherent relationship is disrupted and the individual diffraction event from individual fibrils revealed. Here we made a series of mechanical tests on strips of bovine cornea at extensions well above any physiological limit (up to $692 \%$ of the rest length) in order to reveal the changes in the interfibrillar relationships that may underlie the resting state.

\section{MATERIALS AND METHODS}

\subsection{Materials}

Corneal samples from 6-month old bovine eyes were obtained from a local abattoir and dissected from the scleral tissue leaving approximately $3 \mathrm{~cm}$ diameter discs. All samples were dissected from fresh eyes and stored above saline solution to maintain the correct level of hydration. The specimens were cut into $5 \mathrm{~mm}$ wide strips from the central portion of the cornea (in case the anatomical position was important, all slices were parallel and sagittal) and mounted onto a specially designed stretching rig. The cornea strips were kept fully hydrated using PBS pH7.4 with an ionic strength of $0.15 \mathrm{M} \mathrm{NaCl}$ and subjected to a controlled, incremental strain in the axial direction (up to failure of the sample). Total extension varied between samples due to failure at different strains.

\subsection{Data Collection}

The X-ray diffraction measurements were conducted at the high brilliance synchrotron source at European Synchrotron Radiation Facility (ESRF) in Grenoble, France on beamline ID02 using a 10m sample-to-detector distance. Small-angle X-ray diffraction patterns were collected simultaneously as the cornea strips underwent applied axial strain in a specially designed stretching rig [12]. Samples were bathed in PBS and covered by two thin mica plates to maintain hydration. Incident X-rays were passed through the centre of each cornea in a direction parallel to the optical axis. Images were collected from 16 different cornea samples on three occasions where the number of images collected varied depending on the point at which the sample failed. Typically between 30 and 40 images were collected during each stretching event on each sample using a Thomson X-ray Intensifier (TH 49-427) lens coupled to a FReLoN CCD camera $(2048 \times 2048$ pixels). This detector has an active area of size $180 \mathrm{~mm}$ and a frame rate of 14 images/s $(1024 \times 1024$ pixels $)$, with a 14-bit nominal dynamic range. The delay time between increments of stretch was between 5 and 10 seconds. The exposure time in each case was 0.1 second to minimise dehydration of the cornea. The camera was calibrated using the characteristic $67 \mathrm{~nm}$ meridional spacing of rat tail tendon and the wavelength of the X-rays was fixed at $0.0995 \mathrm{~nm}$. The scattering images presented as a series of five meridional diffraction orders superimposed upon a fibrillar interference function and Bessel function due to the size and lateral disposition of adjacent collagen fibrils.

\subsection{Data Analysis}

X-ray diffraction patterns were analyzed using in house software. The two dimensional detector output was converted into one-dimensional profiles of integrated intensity versus scattering vector $Q$ where

$\mathrm{Q}=4 \pi / \lambda \sin (\theta / 2)$ over 360 degrees of isotropy, al- 
though in some cases where preferred orientation was observed, different aspects of the scattering image could be extracted. The one dimensional profiles were analyzed using the one dimensional peak fitting software Peakfit4 (AISL).

\subsection{Analysis and Identification of Key Reflections}

Each X-ray frame contained information from the axial structure of fibrils - the meridional reflectionsscattering from the cylindrical fibrils, interference functions due to the specific distances between fibrils and in some cases unreported long range reflections. Peakfit4 (AISL) software was used to determine the peak size shapes and integrated intensity of the meridional reflections. Peakfit4 (AISL) was used to obtain the peak maxima and minima of the scattering corresponding to the cylindrical transform of the collagen fibrils, these can be well described as a Bessel function where the first Bessel function minimum occurs at $R=3.83 / \pi D$, where $\mathrm{R}$ is the reciprocal space coordinate of the reflection and $\mathrm{D}$ is the fibril diameter [6].

In addition to calculating the fibril diameter the average centre-to-centre interfibrillar spacing (d) was calculated using $\mathrm{d}=1.12 \mathrm{p}$, where $\mathrm{p}$ is the corresponding intermolecular lateral packing Bragg peak [3,6] and 1.12 is a packing factor that assumes the fibrils are arranged in a hexagonal-like lattice. (a)

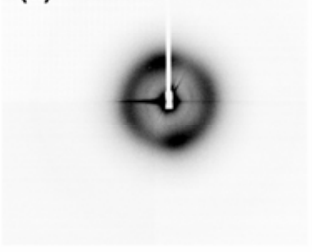

(e) (b)

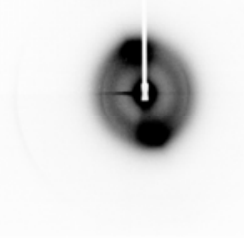

(f)

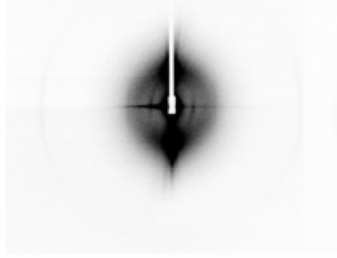

\section{RESULTS}

By using a highly intense X-ray beam and recording at very low scattering angles combined with mechanical stretching changes long range order in corneal fibrils can be revealed to a resolution of approximately $200 \mathrm{~nm}$. Our studied showed that the SAXS (Qmax $=0.06 \mathrm{~nm}^{-1}$, min $=0.005 \mathrm{~nm}^{-1}$ ) pattern of cornea changed significantly with extension and a number of scattering features evolved during extension. We have identified, measured and interpreted the changes observed that speak to changes in interfibrillar interactions.

We have identified, measured and interpreted the changes observed that speak to changes in interfibrillar interactions. Figure 2 shows a gallery of the variety of two-dimensional X-ray diffraction patterns taken from over 400 images of 16 different cornea samples at different stages of mechanical strain. The scattering pattern at resting state shows two main partially superimposed features, firstly a broad peak with a maxim in the region of $\mathrm{Q}=0.013 \mathrm{~nm}^{-1}$ that corresponds to the lateral interfibrillar interference function. The radial intensity distribution of this reflection is indicative of the macroscopic organisation of collagen fibrils and fibrillar lamellae, secondly a series of sharper arced meridional reflections where in the resting state the 3 rd order at $Q=0.045$ $\mathrm{nm}^{-1}$ is prominent. Upon extension, a range of different characteristic features were observed to emerge in the diffraction images, the most important were as below. (c)

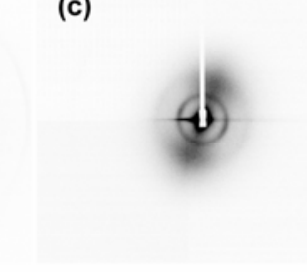

(g)

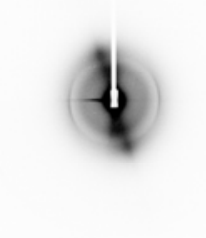

(d)

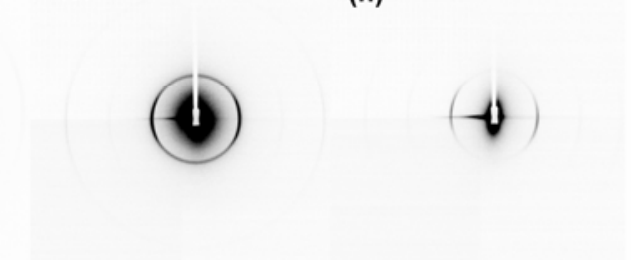

Figure 2. Two-dimensional X-ray diffraction patterns collected from different cornea samples at different stages of mechanical strain. (a) A typical image taken from a cornea with no mechanical strain applied to the cornea, (b) shows an image where the first order meridional reflection of cornea is beginning to be observed after application of mechanical strain. (c) Shows an image where a new reflection has appeared which corresponds to a periodicity of approximately $120 \mathrm{~nm}$. (d) An image where preferential alignment is observed in the meridional reflections. (e) Shows a new reflection corresponding to a periodicity of approximately $120 \mathrm{~nm}$. (f) Shows the presence of two distinct interfibrillar reflections corresponding to different interfibrillar distances. $(\mathrm{g})$ and $(\mathrm{h})$ show a more pronounced and visible first order meridional reflection of cornea. 


\subsection{Emergence of the First Order Meridional Reflection}

During strain extensions to breakage-up to $692 \%$ percent in the highest case, and in some cases before the sample failed macroscopically, a strong first order of meridional diffraction peak was observed, this is in addition to the third order that is usually the most prominent meridional diffraction in the resting state.

The position and breadth of the refection with respect to scattering vector $\mathrm{Q}$, and therefore the axial lattice structure within the fibrils did not change sensibly. Example X-ray scattering patterns exhibiting this feature are shown in Figures 2(d), and (f)-(h) there are at extensions of $80 \%, 558 \%, 650 \%$ and $692 \%$ of the rest length respectively within different samples.

These clearly show the emergence of a first order diffraction peak at $\mathrm{Q}=0.015 \mathrm{~nm}^{-1}$ that is visible above the lateral interfibrillar scattering pattern at $\mathrm{Q}=0.014 \mathrm{~nm}^{-1}$. The observation of the first order of meridional diffracttion at the highest levels of extension observed (over $500 \%$ ) was combined with a much decreased peak corresponding the interfibrillar interference scattering indicating a decoupling and loss of spatial regularity between the fibrils both axially and laterally (Figures 2(g) and (h)).

A corresponding one-dimensional linear trace for cornea upon application of mechanical strain within a single cornea is shown in Figure 3.

Figure 3(a) shows the radially averaged scattering profile from the rest state cornea with no mechanical strain applied. Figures 3(b) and (c) shows the emergence of a first order reflection upon application of mechanical strain at $233 \%$ and $675 \%$ the rest length and the loss of the lateral interfibrillar interference peak. The presence of the first order reflection is visible at a position of $0.015 \mathrm{~nm}^{-1}$ which corresponds to an axial-period of 66.6 $\mathrm{nm}$ this value does not change during the mechanical testing as judged by the movement of the 3rd meridional order which is visible in all samples. The scattering profile in Figures 3(a) and (b) also shows the broad lateral interfibrillar interference function at $0.013 \mathrm{~nm}^{-1}$ and $0.014 \mathrm{~nm}^{-1}$ respectively. This movement possibly confounds the appearance of a weak first order in the resting state. The appearance and minimally altered position of higher orders of the meridional diffraction series demonstrates that the collagen axial periodicity within a fibril remained.

\subsection{An Increase in the Interfibrillar Spacing}

During the application of mechanical strain, the observed fibril diameter of the bovine cornea strip samples remained at $38.5 \mathrm{~nm}( \pm 1.5 \mathrm{~nm})$ this was determined by the approximation of the scattering corresponding to the lateral fibrillar interaction curve to that of a Bessel Function convolved with an interference function, which is appropriate for physiological hydrated bovine cornea $[4,13]$.

The position of the scattering maxima and minima of the cylindrical function did not change sensibly upon extension until in some cases the lateral interference

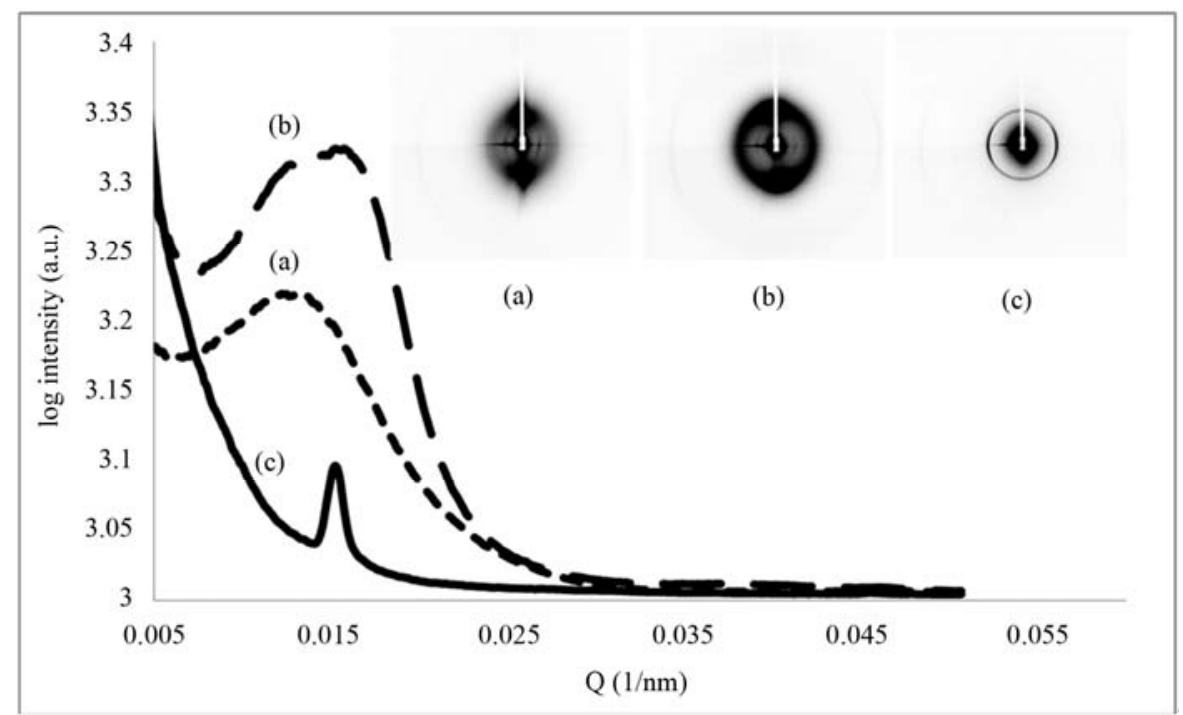

Figure 3. One-dimensional small-angle X-ray scattering curves for cornea upon application of mechanical strain and the corresponding two-dimensional small-angle X-ray scattering patterns. (a) Shows the rest state cornea with no mechanical strain applied. (b) Shows the emergence of a first order reflection upon application of mechanical strain. (c) Shows the loss of the interfibrillar separation peak and the presence of a first order reflection at 0.015 $\mathrm{nm}^{-1}$. 
function rapidly disappeared between exposures, however the meridional peaks remained. In many cases of macroscopic sample extension, we observed that the peak of the interfibrillar interference function moved to a lower Q value as the cornea strips are strained (see Figure 4), this corresponds to an increase in interfibrillar separation of the collagen fibril whilst the diameter of invidividual fibrils remain constant.

On average the interfibrillar spacing increased in 11 of the 16 samples tested from rest state to over $250 \%$ extension, this type of behaviour is indicative of an auxetic behavior at the interfibrillar level (see Figure 5).

\subsection{An Appearance of a New Periodicity}

The diffraction patterns of extended samples at very low $Q$ values affected by the beamline geometry reveal an azimuthally broad peak at $120 \mathrm{~nm}$ (see Figure 6) not been previously reported.

This feature is only observed upon extension and is unseen in the relaxed samples. The peak is observed at different stages of extensions in five of the 16 cornea samples tested. The full width at half maximum of the newly observed peak was between $40 \%$ and $60 \%$ broader than the meridional peaks due to fibrillar collagen therefore indicating it is from a different molecular assembly.

\section{DISCUSSION}

The structural studies described here reveal features and phenomena when corneal tissue is perturbed to breaking point. In most studies of collagen rich tissues, structure determination and mechanical modelling regard the fibrillar structures as discrete entities with no coherent relationship between fibrils. The possible evidence of a coherent spatial relationship between fibrils comes from evidence of fibril fusing, which requires an axial spatial alignment of the fibrils that fuse so that their gap and overlaps are matched. Also in mineralised tissues such as turkey tendon, there is evidence that there is a coherent deposition of mineral between and within adjacent fibrils [1], these however both indicates fibril register without a specific axial displacement.

In the experiments conducted here, the enhanced diffraction from the first order is commensurate with the debonding of coherent fibrillar interactions that have held local interactions in a specific axially displaced alignment. A similar occurrence was found in fibrillin rich microfibrils where X-ray diffraction predicted a significant population of the fibrils were associated in a specific manner that involved an average relative offset of 0.33 of the fundamental periodicity along each fibril [12].

The changes in interfibrillar distances especially the increases in the interfibrillar distance as a function of strain is characteristic of an auxetic material, and can possibly be explained by a series of sacrificial adjustments being made by the interfibrillar material as the fibrils may begin to slide past each other. One theory is that there is an arrangement of proteoglycan arms surrounding the collagen fibril maintaining a specific order to the fibrils. It has been proposed that it would be possible for collagen fibrils in corneal stroma to touch if

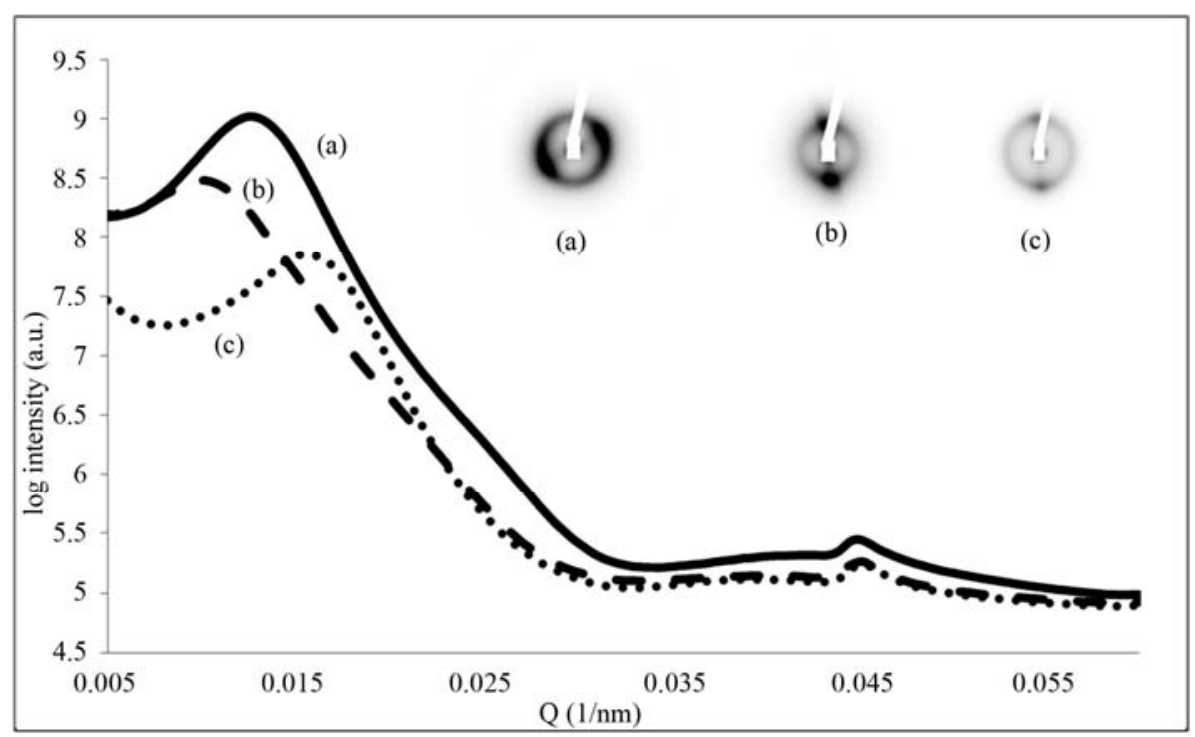

Figure 4. One-dimensional small-angle X-ray scattering curves for cornea upon application of mechanical strain and the corresponding two-dimensional small-angle X-ray scattering patterns. (a) Shows the rest state cornea with no mechanical strain applied. (b) The interfibrillar separation moves to a lower Q value upon application of mechanical strain. (c) The interfibrillar separation moves to a higher $\mathrm{Q}$ value upon further application of mechanical strain. 

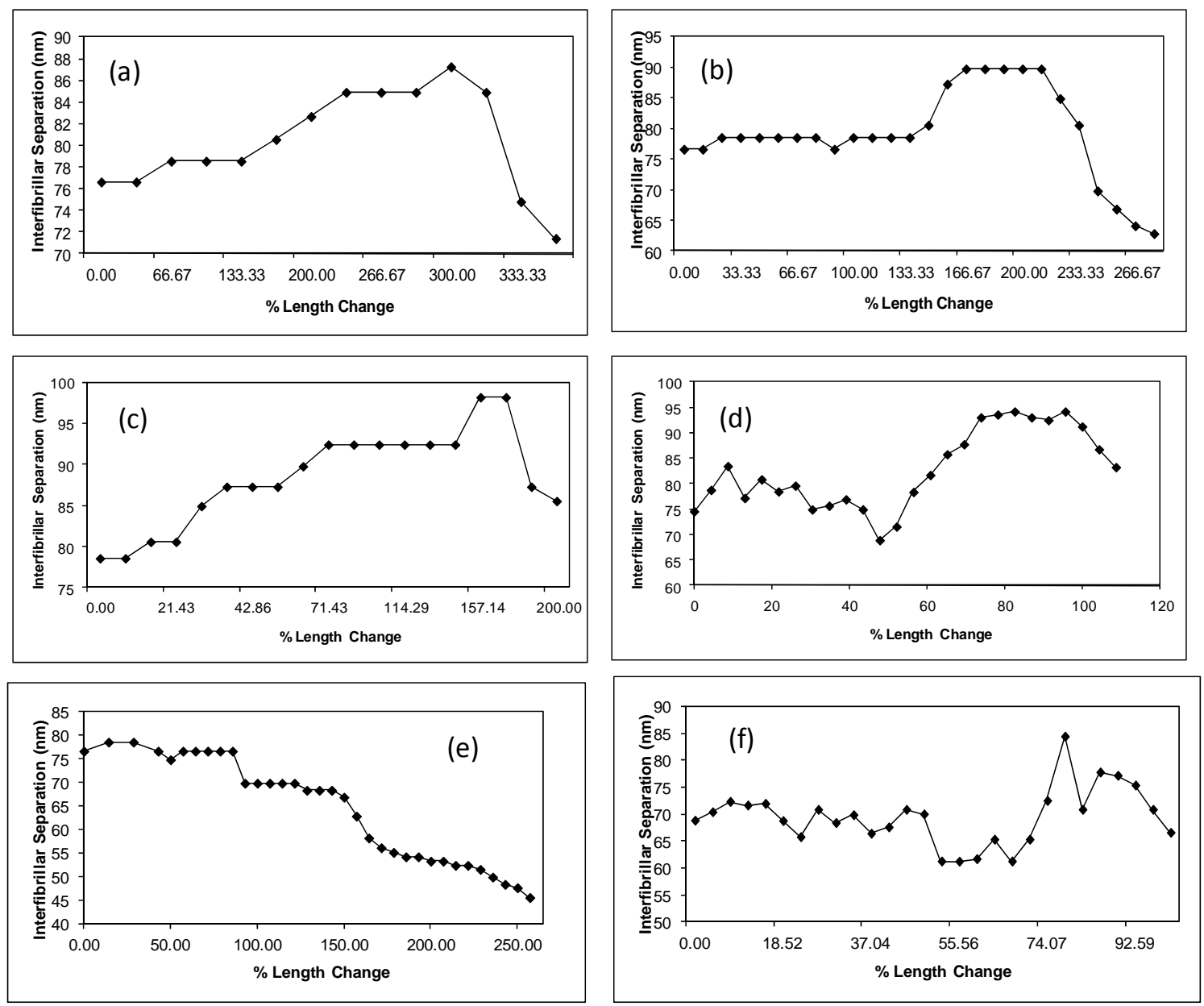

Figure 5. The interfibrillar separation as a function of \% length change for six of the 16 cornea samples that were axially strained. In (a) to (d) an increase in interfibrillar separation was observed followed by a decrease in interfibrillar separation before failure. (a) $76.62 \mathrm{~nm}$ to $87.27 \mathrm{~nm}$ to $71.40 \mathrm{~nm}$, (b). $76.78 \mathrm{~nm}$ to $89.76 \mathrm{~nm}$ to $62.83 \mathrm{~nm}$, (c) 78.54 $\mathrm{nm}$ to $98.17 \mathrm{~nm}$ to $85.53 \mathrm{~nm}$, (d) $68.9 \mathrm{~nm}$ to $94.19 \mathrm{~nm}$ to $83.26 \mathrm{~nm}$. In (e) and (f) no initial increase was observed in the interfibrillar separation only a decrease in interfibrillar separation.

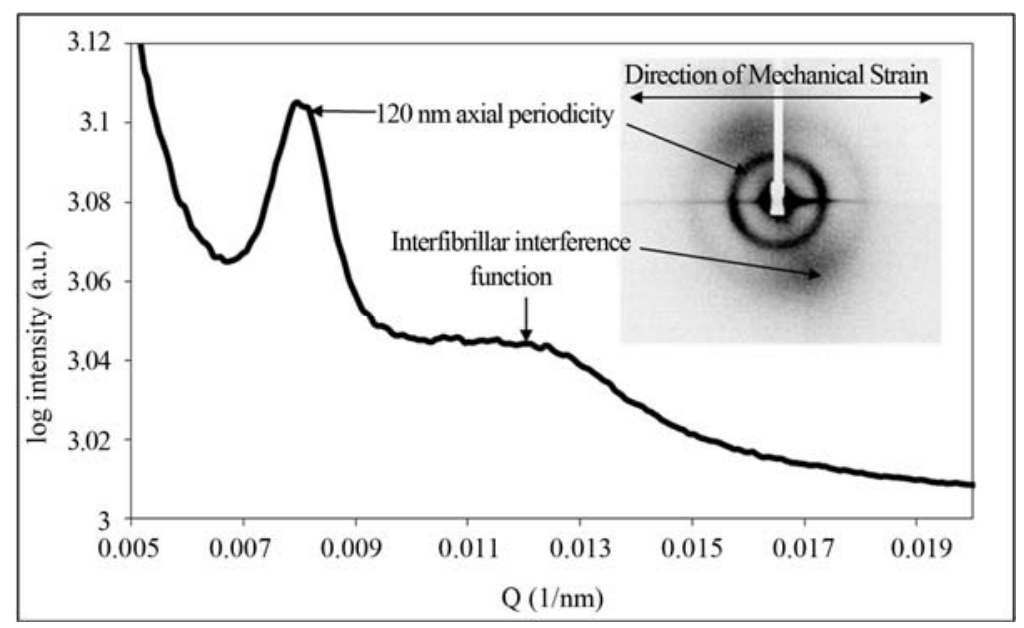

Figure 6. Small-angle X-ray scattering pattern and corresponding linear trace from cornea showing the presence of an additional reflection corresponding to an axial period of approximately $120 \mathrm{~nm}$. Also highlighted are the inter-fibrillar interference function and the direction in which the axial strain was applied. 
they are free to move in a matrix of water and proteoglycans [3].

The source of the $120 \mathrm{~nm}$ reflection is possibly due to the alignment of Type VI collagen which is present at high levels in the corneal matrix [14]. The role of this material is far less well understood and studies compared to the bulk Type I/V heterotypical fibrils of the corneal lamella. The cornea is predominantly Type I collagen but Type VI collagen has also been found in cornea as a bead filament structure with a characteristic axial periodicity of approximately $100-120 \mathrm{~nm}$. Some studies have suggested that the role of Type VI collagen is to maintain and restrict movement of collagen fibrils relative to one another [14]. Not all the phenomena have a related physicochemical basis and the $120 \mathrm{~nm}$ peak may be an unrelated ordering event of a different collagen class in the cornea.

\section{CONCLUSION}

In this study, we demonstrated that the local organisation between fibrils may be essential to suprafibrillar organisation of collagen. In disrupting the specific suprafibrillar relationship between fibrils and the coherent staggered axial relationship between collagen fibrils was revealed. In previous mechanical and structural models the suprafibrillar organisation in collagen has not been considered, however this work has demonstrated the potential need to consider the specific suprafibrillar relationship between fibrils.

\section{ACKNOWLEDGEMENTS}

We are grateful to T. Narayanan and T. Weiss at beamline ID02, at the European Synchrotron Radiation Facility (ESRF) for user support.

\section{REFERENCES}

[1] Traub, W., Arad, T. and Weiner, S. (1992) Growth of mineral crystals in turkey tendon collagen fibers. Connect Tissue Research, 28, 99-111. doi:10.3109/03008209209014230

[2] Wess, T.J., Purslow, P.P., Sherratt, M.J., Ashworth, J., Shuttleworth, C.A. and Kielty, C.M. (1998) Calcium Determines the supramolecular Organization of Fibrillinrich Microfibrils. The Journal of Cell Biology, 141, 829837. doi:10.1083/jcb.141.3.829

[3] Fratzl, P. and Daxer, A. (1993) Structural transformation of collagen fibrils in corneal stroma during drying-An
X-ray scattering study. Biophysical Journal, 64, 12101214. doi:10.1016/S0006-3495(93)81487-5

[4] Maurice, D.M. (1957) The structure and transparency of the cornea. The Journal of Physiology, 136, 263-286

[5] Meek, K.M. and Leonard, D.W. (1993) Ultrastructure of the corneal stroma: A comparative study. Biophysical Society, 64, 273-280. doi:10.1016/S0006-3495(93)81364-X

[6] Scott, J.E. and Thomlinson, A.M. (1998) The structure of interfibrillar proteoglycan bridges (shape modules) in extracellular matrix of fibrous connective tissues and their stability in various chemical environments. Journal of Anatomy, 192, 391-405. doi:10.1046/j.1469-7580.1998.19230391.x

[7] Wall, R.S., Elliott, G.F., Gyi, T.J., Meek, K.M. and Branford-White, C.J. (1988) Bovine corneal stoma contains a structural glycoprotein located in the gap region of the collagen fibrils. Bioscience Reports, 8, 77-83. doi:10.1007/BF01128974

[8] Hodge, A.J. and Petruska, J.A. (1963) Recent studies with the electron microscope on ordered aggregates of the tropocollagen molecule. In: Ramachandran, G.N., Ed., Aspects of Protein Structure, Academic Press, New York, 289-300.

[9] Scott, J.E. and Haigh, M. (1985) Proteoglycan-type I collagen fibril interactions in bone and non-calcifying connective tissues. Bioscience Reports, 5, 71-81. doi:10.1007/BF01117443

[10] Meek, K.M., Elliott, G.F. and Nave, C. (1986) A synchrotron X-ray diffraction study of bovine cornea stained with cupromeronic blue. Collagen Related Research 6, 203218. doi:10.1016/S0174-173X(86)80026-7

[11] Wess, T.J., Purslow, P.P. and Kielty, C.M. (1998) X-ray diffraction studies of fibrillin-rich microfibrils: Effects of tissue extension on axial and lateral pack. Journal of Structural Biology, 122, 123-127. doi:10.1006/jsbi.1998.3992

[12] Holmes, D.F., Gilpin, C.J., Baldock, C., Ziese, U., Koster, A.J. and Kadler, K.E. (2001) Corneal collagen fibril structure in three dimensions: Structural insights into fibril assembly, mechanical properties, and tissue organisation. Proceedings of the National Academy of Sciences, 98, 7307-7312.

[13] Kern, P., Menasche, M. and Robert, L. (1991) Relative rates of biosynthesis of collagen type I, type $\mathrm{V}$ and type VI in calf cornea. Biochemistry, 274, 615-617.

[14] Keene, D.R., Lunstrum, G.P., Morris, N.P., Stoddard, D.W. and Burgeson, R.E. (1991) Two type XII-like collagens localize to the surface of banded collagen fibrils. The Journal of Cell Biology, 113, 971-978. doi:10.1083/jcb.113.4.971 